\title{
STABILITY BOUNDARY AND DESIGN CRITERIA FOR HAPTIC RENDERING OF VIRTUAL WALLS
}

\author{
Thomas Hulin ${ }^{*, 1}$ Carsten Preusche* \\ Gerd Hirzinger* \\ * Institute of Robotics and Mechatronics, DLR (German \\ Aerospace Center), D-82234 Wessling, Germany, \\ Thomas.Hulin@dlr.de
}

\begin{abstract}
This paper is about haptic simulations of virtual walls, which are represented by a discrete PD-control. A normalized discrete-time transfer function is used to derive the fundamental stability boundaries for this problem. Hereby, the case of direct action and the more often case of an one sampling step delayed action are addressed. Inside the stable region the set of all parameters was determined that result in real system poles. Furthermore, three different design criteria are compared to find optimum control parameters for the virtual wall. Finally, important conclusions for haptic simulations are derived.

Copyright (c) 2006 IFAC
\end{abstract}

Keywords: Haptic Rendering, Virtual Spring, Virtual Damper, PD-Control, Stability Boundary, Virtual Wall

\section{INTRODUCTION}

The use of haptic interfaces can be divided into two different application fields, namely teleoperation and virtual haptic simulations. For both tasks, energy leaks occur due to time delay of signal transmission and finite sampling rate. If the energy generated is greater than the mechanical dissipation, instabilities will occur that reduce transparency of haptic simulations or, at worst, may injure the operator. Thus, guaranteeing stability is crucial for these application tasks.

A common way of guaranteeing stability for virtual haptic simulations is to ensure passivity for the haptic device (Colgate and Brown, 1994). Although this is a very general approach, it has the disadvantage that it is conservative and requires the presence of mechanical damping. For mechan-

\footnotetext{
1 This work was partly supported by the Volkswagen AG
}

ically undamped systems with one sampling step time delay, Salcudean and Vlaar (Salcudean and Vlaar, 1997) obtained the exact stability conditions by transforming the continuous-time behavior of the haptic device via an approximationformula into the discrete-time domain. They defined a dimensionless "damping" and a respective dimensionless "stiffness" and thereby computed the stability boundaries.

Recently, Gil et al. (Gil et al., 2004) computed the exact stability boundary for virtual damping and stiffness for their haptic interface. Furthermore, they showed that a human operator tends to stabilize the system, i.e. the worst-case scenario for stability is the situation when the operator is not grabbing the haptic interface.

In this paper, an exact transformation of the (impedance-) control loop of a virtual haptic simulation into the discrete-time domain is performed to compute the fundamental stability boundaries 


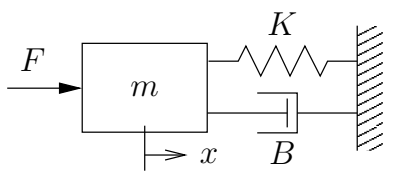

Fig. 1. The haptic interface modeled as a simple mass $m$ combined with a virtual spring and damper.

for the worst case, where a human operator is not taken into account. Hereby, the case of direct action and the more often case of an one sampling step delayed action are addressed. In section 6 three different design criteria for control rules are compared. Finally, in section 8 important conclusions for haptic simulations are derived.

One of the main outcomes of this paper is, that these curves determined hold for all haptic interfaces where mechanical damping can be neglected (in contrast to the discrete damping).

\section{PROBLEM DESCRIPTION}

In virtual force-feedback simulations stiff walls are often modeled as spring-damper systems. To generate a realistic impression of hard contacts, the stiffness of virtual surfaces must be at least $K=2000 N / m$ (Massie and Salisbury, 1994) if a PHANToM is used as haptic interface. However, it is not possible to increase stiffness arbitrarily. A higher stiffness causes a higher energy gain (Basdogan and Srinivasan, 2001) that tends to destabilize the system. Furthermore, the motors and the mechanical structure of the haptic device are limiting the maximum displayable stiffness. For obtaining stability, collisions with stiff walls are often represented by a stiff spring $K$ together with a damper $B$, that has the purpose of dissipating the energy generated and guaranteeing passivity (see Fig. 1). This spring-damper system is equivalent to a discrete PD-controller.

Many haptic interfaces, e.g. the PHANToMdevice, were designed with low mechanical damping, as one prerequisite for transparency of haptic simulations is that "free space must feel free" (Massie and Salisbury, 1994). Thus, in this paper only systems with no mechanical damping are considered.

For impedance control the worst-case scenario for stability is the situation when the operator is not grabbing the haptic device (R. J. Adams and B. Hannaford, 1999; Gil et al., 2004). As the haptic device has to be stable for all possible configurations, the stability analysis was performed for this worst-case scenario without a human operator: $F_{H}=0$.

A time delay $L$ from the measured position to the performed force might occur due to various rea-

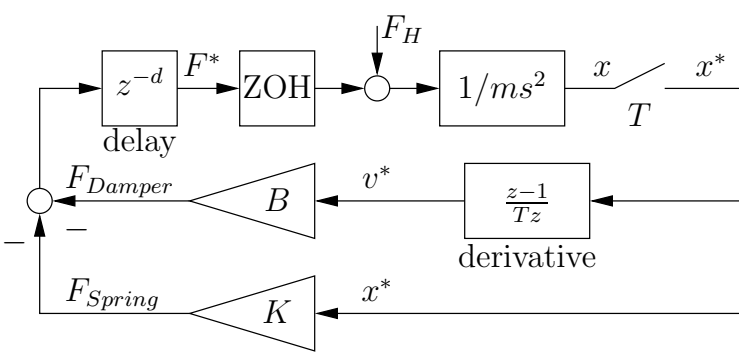

Fig. 2. Control loop with continuous-time mass $m$ and no mechanical damper.

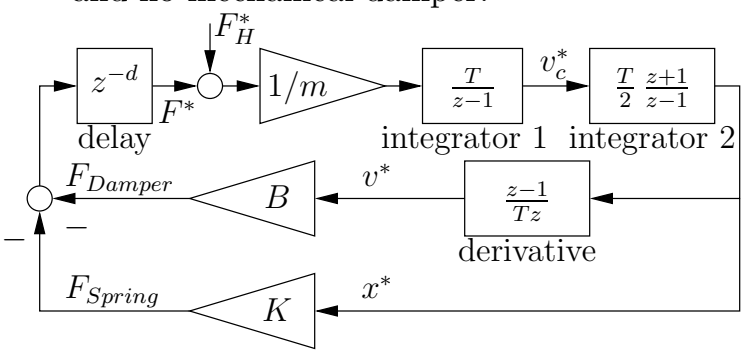

Fig. 3. Discrete-time control loop which is equivalent to the system shown in Fig. 2.

sons, e.g. the time needed for the force calculation of complex virtual scenarios. Yet, in most publications about this topic this delay is usually omitted (Gil et al., 2004), (Colgate and Brown, 1994) or assumed to be one sampling period $T$ (Salcudean and Vlaar, 1997). In the following both of these cases are analyzed. Though, a general transfer function has to be found first.

\section{DISCRETE-TIME CONTROL LOOP}

With the assumptions of the previous section, the control loop of a haptic simulation can be set up as illustrated in Fig. 2. The time delay is implemented as discrete delay with the delay factor $d=L / T$, where $L$ is the time delay and $T$ is the sampling time. As described above, the two most common cases are addressed in this paper:

$\mathbf{d}=\mathbf{0}$ : direct (undelayed) action

$\mathbf{d}=\mathbf{1}$ : one sampling step delayed action

For analyzing the system concerning stability, the continuous-time integrators have to be transformed into the discrete-time domain. Standard methods for this transformation are Euler's and Tustin's numerical approximations. However, they are approximations and the result would not be exact. Yet, the cascade of these two approximations result in the exact discrete-time equivalent (see Fig. 3).

Integrator 1 integrates the acceleration, which is constant over one sampling period $T$ under the condition that no mechanical damping exists. Therefore, the resulting velocity $v_{c}^{*}$ of the mass is linear over one sampling period $T$. In conclusion, the appropriate transfer function to compute the 


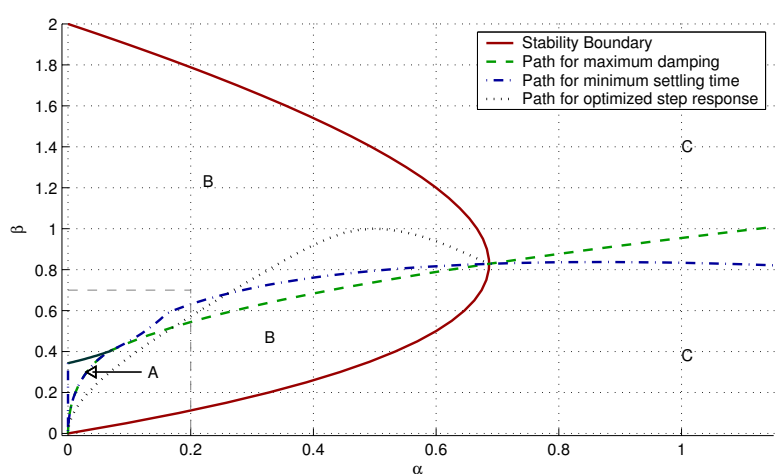

Fig. 4. The stable $(A \cup B)$ and unstable $(C)$ region in the $(\alpha, \beta)$-plane for the case $d=0$. In region $A$ are all three poles real, in $B$ is one pole-pair complex.

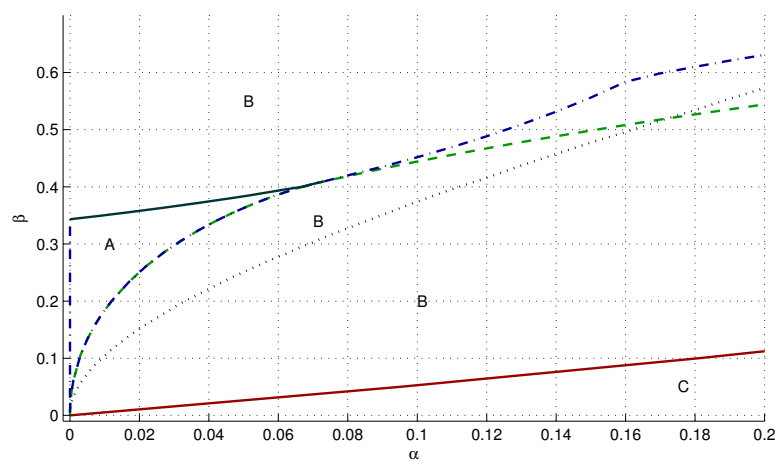

Fig. 5. Magnified region of Fig. 4.

position $x^{*}$ out of $v_{c}^{*}$ is given by integrator 2 . Notice, that the velocity $v^{*}$ has a phase shift of $T / 2$ compared to $v_{c}^{*}$.

From Fig. 3, the characteristic equation can be easily computed to be

$0=K T^{2} z(z+1)+B T\left(z^{2}-1\right)+2 m z^{1+d}(z-1)^{2}$.

\section{NORMALIZED STIFFNESS AND DAMPING}

Considering the characteristic equation (1), it becomes apparent that the virtual stiffness $K$ is proportional to $m / T^{2}$, and that the virtual damping $B$ is proportional to $m / T$. By substituting normalized variables for the stiffness $K$ and the damping coefficient $B$ the characteristic equation (1) becomes independent of the physical system parameters $m$ and $T$

$$
\begin{aligned}
& \alpha=K \cdot T^{2} / m \\
& \beta=B \cdot T / m .
\end{aligned}
$$

The normalized characteristic equation follows as

$$
0=\alpha z(z+1)+\beta\left(z^{2}-1\right)+2 z^{1+d}(z-1)^{2} .
$$

For $d=1$, this equation corresponds to the normalized characteristic equation found by Salcudean and Vlaar (Salcudean and Vlaar, 1997).

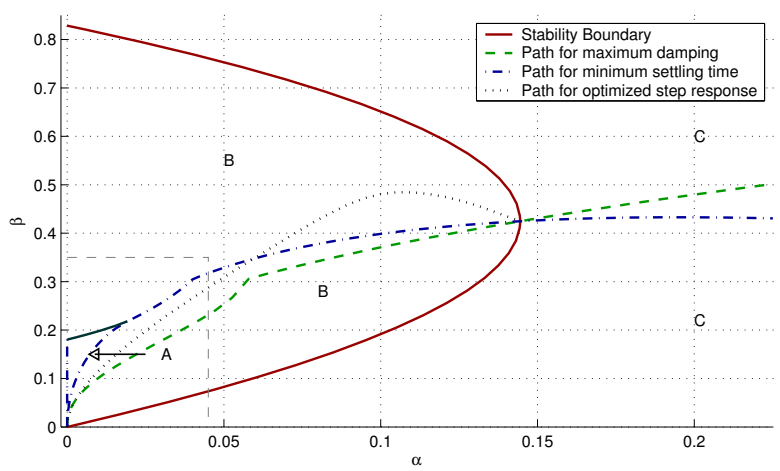

Fig. 6. The stable $(A \cup B)$ and unstable $(C)$ region in the $(\alpha, \beta)$-plane for the case $d=1$. In region $A$ are all four poles real, in $B$ is one pole-pair complex.

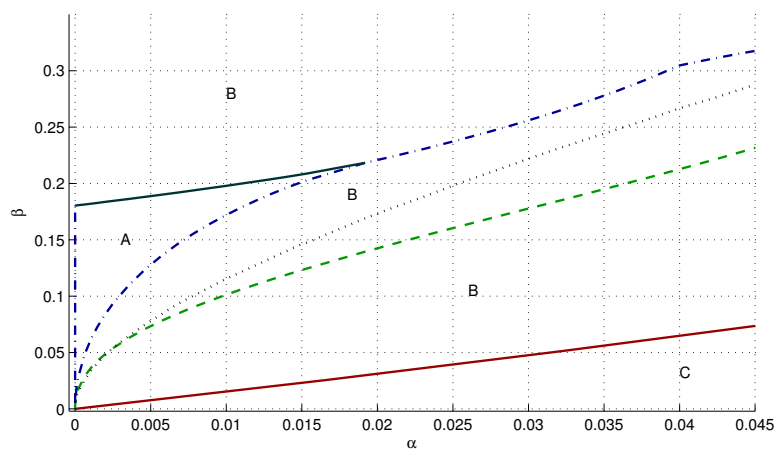

Fig. 7. Magnified region of Fig. 6.

Also, the zeros of the normalized transfer function are independent of the mass and the sampling time. These parameters occur only as factor in the numerator. For the normalized transfer function $G(z)$ from force $F_{H}^{*}$ to position $x^{*}$ yields

$$
G(z)=\frac{z^{1+d}(z+1) T^{2} / m}{\alpha z(z+1)+\beta\left(z^{2}-1\right)+2 z^{1+d}(z-1)^{2}}
$$

\section{STABILITY BOUNDARIES}

Using the normalized characteristic equation (3), it is possible to plot the stability boundary of the system in the $(\alpha, \beta)$-plane. The system is stable if all poles lie inside the unit circle in the $z$-plane. As the order of the characteristic polynomial in (3) is $3+d$, the roots had to be calculated iteratively for $d>0$.

For the two cases $d=0$ and $d=1$, the results are shown in Fig. 4-7. Curves, similar to parabolas, are dividing the stable $(A \cup B)$ from the unstable $(C)$ regions. Inside region $A$ all poles are real, i.e. no oscillations occur.

Exact equations of the stability boundaries would be useful for several purposes. Yet, as the stability boundaries were computed iteratively, only approximations can be stated. Good polynomial approximations for the shown boundaries could 
be found by computing the polynomial factors out of some of the iteratively determined points. For $d=0$ yields

$$
\begin{aligned}
\alpha_{d=0}(\beta)= & 2 \beta-1.984 \beta^{2}+0.924 \beta^{3}-0.35 \beta^{4} \\
& +0.0865 \beta^{5}-0.00975 \beta^{6}
\end{aligned}
$$

and for $d=1$

$\alpha_{d=1}(\beta)=\frac{2}{3} \beta-0.7425 \beta^{2}-0.048 \beta^{3}-0.03278 \beta^{4}$.

The absolute error for these approximations to the exact stability boundaries is less than $\triangle \alpha=$ 0.0003 for (5) and less than $\triangle \alpha=0.0001$ for (6).

Remark 1. The results correspond to those of Gil et al. (Gil et al., 2004), where, among other things, an approximated formula for the region around the origin is presented, that fits the following condition for stability gained by linearization of the stability boundary for $d=0$ around zero

$$
\alpha(\beta)<2 \beta \text {. }
$$

Linearizing the stability boundary for $d=1$ around zero yields

$$
\alpha(\beta)<2 / 3 \beta .
$$

Remark 2. The exact value-pair $[\alpha, \beta]$ for the maximum achievable stiffness for which the system is stable with no delay $d=0$ can be calculated exactly to be $[12-8 \sqrt{2},-2+2 \sqrt{2}] \approx$ $[0.6863,0.8284]$. For $d=1$ it was not possible to calculate the exact value pair, but the iteratively determined values are [0.144469, 0.424694]. For zero stiffness $\alpha=0$ the exact values for the maximum achievable damping are $\beta=2$ for $d=0$, and $\beta=-2+2 \sqrt{2} \approx 0.8284$ for $d=1$.

\section{CONTROL RULE}

During most haptic simulations the virtual stiffness varies considerably, and it would be valuable to have a rule that defines the optimal values for the virtual damping dependent on the particular stiffness values. As the parameters $T$ and $m$ are only a factor of the transfer function (4), it is possible to give an optimal control rule for all haptic devices with negligible mechanical damping. Yet, there are different solutions depending on the optimization criterion.

This section discusses three design criteria for this problem, one by maximizing the system damping, one by minimizing the settling time and one by minimizing the square error of a step response.

\subsection{Path for Maximum Damping}

Computing the system damping of discrete-time systems is performed by drawing a logarithmic
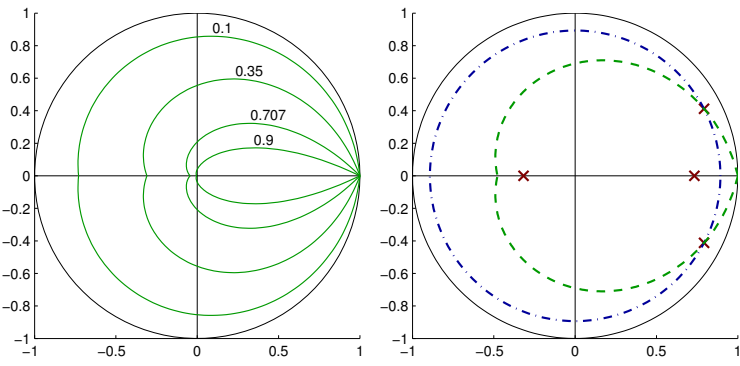

Fig. 8. Left: The spirals inside which all system poles must lie to provoke a system damping of $D=[0.1,0.35,0.707,0.9]$. Right: The system poles for $d=1, \alpha=0.075$ and $\beta=0.371$. The resulting damping is $D=0.229$ and the radius of the circle is 0.893 .

spiral around all system poles (see Fig. 8 left). For smaller spirals the damping is increasing. If the stiffness $\alpha$ is gridded, for each $\alpha$-value a $\beta$ can be determined where the system damping is maximum. The result is a path in the $[\alpha, \beta]$ plane. This path for maximum damping is shown as dashed lines in Fig. 4-7.

\subsection{Path for Minimum Settling Time}

The settling time of discrete-time systems depends mainly on the largest eigenvalue $|z|_{\max }$ (Franklin et al., 1998). The normalized damping coefficients $\beta$ were computed for gridded values of $\alpha$, such that all poles of the normalized characteristic equation (3) lie inside a circle with minimum radius (see Fig. 8 right). The resulting dashdotted lines are shown in Fig. 4-7. These curves can be used as an approximation of the path with minimum settling time.

It is interesting that on the first part on this path the system poles are real ${ }^{2}$. In fact, this interval of the path is the lower border of the region $A$ where all poles are real, i.e. below that path one pole-pair becomes complex. The upper boundary of region $A$ is also plotted in Fig. 4-7.

When moving along these paths for minimum settling time and plotting the absolute values of the largest eigenvalue $|z|_{\max }$ it comes apparent that there is a global minimum on the computed curves inside the stability region (see Fig. 9) at:

$\mathbf{d}=\mathbf{0}: \alpha=0.070, \beta=0.405$

$\mathbf{d}=1: \alpha=0.0191, \beta=0.218$

This minimum coincides with the rightmost point of region $A$.

\footnotetext{
${ }^{2}$ For $d=0: \alpha \in[0,0.702]$; for $d=1: \alpha \in[0,0.0191]$
} 

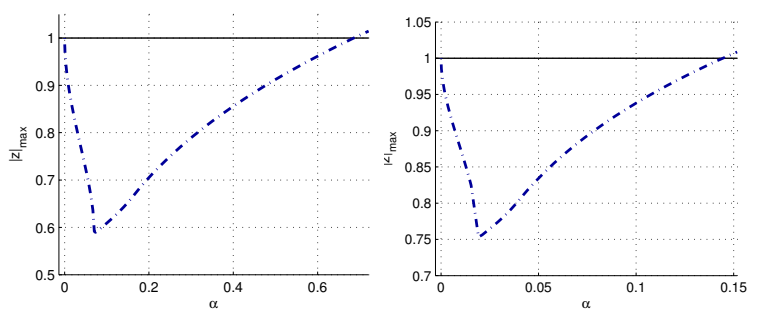

Fig. 9. The absolute value of the maximum pole $|z|_{\max }$ over $\alpha$ plotted along the path for minimum settling time.
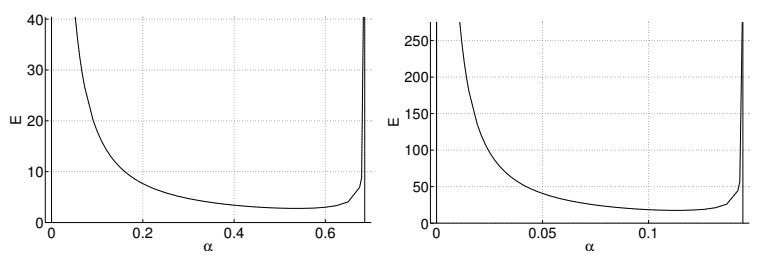

Fig. 10. The square error $E$ for $T^{2} / m=1$ (left: $d=0$, right: $d=1$ ).

6.3 Path for Minimum Square Error of the Step Response

The two paths above were determined solely out of the poles of the transfer function (4). By considering the step response, the zeros are also taken into account. As the final position $x_{r e f}$ of the mass $m$ can be computed very easily via equation (2) and $F_{\text {Spring }}=K x^{*}$, a sensible rule is to minimize the square distance between the step response $x_{\text {step }}$ and the final position $x_{r e f}$, i.e. minimize

$$
E=\sqrt{\sum_{k=0}^{\infty}\left(x_{r e f}(k)-x_{s t e p}(k)\right)^{2}}
$$

The resulting paths are also plotted in Fig. 47 as dotted lines. The first parts of these paths lie below the path for minimum settling time, whereas the right parts are above.

Along these paths the error $E$ is plotted in Fig. 10. The resulting curve has a minimum at:

$\mathbf{d}=\mathbf{0}: \alpha=0.54, \beta=0.99$

$\mathbf{d}=\mathbf{1}: \alpha=0.11, \beta=0.48$

Yet, these minima do not result in good transient responses (compare Fig. 12). Plotting the normalized error $E / x_{\text {ref }}$ yields in another curve (see Fig. 11) whose minima result in faster responses:

$\mathbf{d}=\mathbf{0}: \alpha=0.41, \beta=0.93$

$\mathbf{d}=\mathbf{1}: \alpha=0.09, \beta=0.46$

The step responses of the system at the minima of the path for minimum square error is shown in Fig. 12. The system parameters $m$ and $T$ influence only the scaling of the responses in $x$-direction.
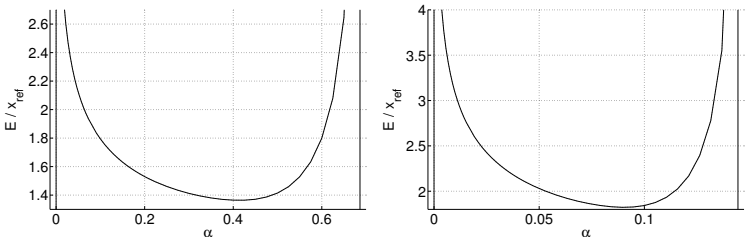

Fig. 11. The normalized square error $E / x_{\text {ref }}$ (left: $d=0$, right: $d=1)$.
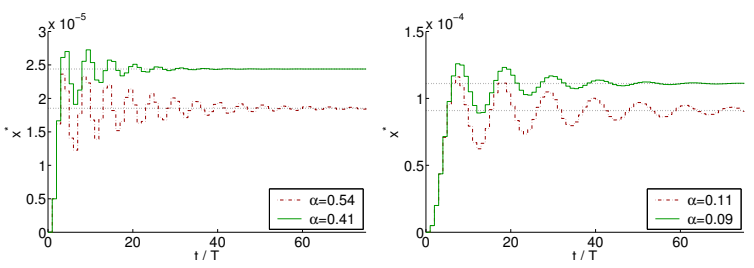

Fig. 12. The step responses for the system with $T=0.001, m=0.1$ at the minima of the path for minimum square error (left: $d=0$, right: $d=1)$.

\subsection{Discussion}

Above, three different design criteria for control rules were presented. But which of them is the most suited rule for haptic rendering? To answer this question the step responses of the system on the three paths are considered. Exemplary, Fig. 13 shows for $d=1$ the responses for two different cases of $\alpha$ :

$\alpha=0.025$ : The path for minimum square error is below the path of minimum settling time. $\alpha=0.08$ : The path for minimum square error is above the path of minimum settling time.

For both cases the response for the values on the path of maximum damping is the worst, as they result in the highest overshoot and in the slowest transient response. For the other two paths the respective response with the less damping is faster for the first few steps but result in a higher overshoot. To compare the impression of these responses, an evaluation with human operators has to be performed.

\section{EXAMPLE WITH THE PHANTOM}

For a haptic device similar to the PHANToM device described in (Massie and Salisbury, 1994), the moving mass and the sampling time are assumed to be $m=0.1 \mathrm{~kg}$ and $T=0.001 \mathrm{~s}$. Furthermore, the case $d=1$ will be considered, i.e. the computed forces are actuated one sampling step after the position is measured. For the optimum controller with respect to the normalized square error of the step response (section 6.3) yields

$$
\begin{aligned}
& K=0.09 \mathrm{~m} / T^{2}=9 \mathrm{kN} / \mathrm{m} \\
& B=0.46 \mathrm{~m} / T=4.6 \mathrm{Ns} / \mathrm{m}
\end{aligned}
$$



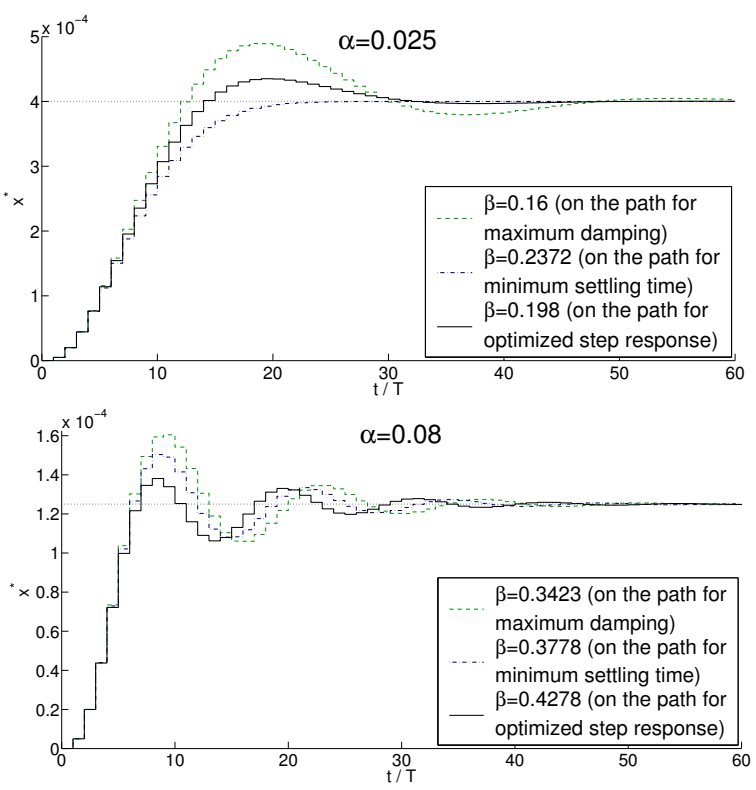

Fig. 13. Three step responses for the system with $T=0.001, m=0.1$ and $d=1$ on the three paths for $\alpha=0.025$ and $\alpha=0.08$.

\section{CONCLUSIONS AND FUTURE WORK}

This article determines the fundamental stability boundaries for the haptic simulation of a virtual wall. Moreover, three different design criteria were compared to find optimum control parameters for the virtual wall. The most evident approximation was the neglection of the mechanical damping.

But for most haptic devices this approximation is insignificant: considering the stability boundary from (Gil et al., 2004) determined for their haptic device under presence of mechanical damping, it fits nearly perfectly the stability boundary determined above (the error at its maximum is less than 1\%).

The following conclusions can be derived out of the results stated above:

Dependency on the sampling time: The maximum achievable stiffness depends quadratically on the sampling rate (see equation (2)). Thus, increasing the sampling rate yields in a much better performance (if other time delay is small compared to the sampling time).

Dependency on the human mass: Haptic devices are often built with little mass at the end effector. Thus, the mass of the human hand or arm strongly influences the effective mass of the haptic device. To achieve a good controller it is inevitable to determine the effective mass of the end-effector of the haptic device, i.e. the mass of the end-effector together with the mass of the human. Another apparent solution would be to develop a haptic device with a big mass at the end-effector, so that the effect of the human hand's mass is small. Yet, this is problematic because of the additional inertia that is disturbing the haptic impression and because of limits in the motors of the haptic device.

Dependency on the mass: Equation (2) indicates a linear dependency of the virtual stiffness and damping on the mass $m$. Considering the stiffness, it is obvious that if doubling the mass, the stiffness has to be doubled, too, to achieve the same system behaviour. However, the higher stiffness does not lead to a better (i.e. faster) behaviour, as the mass is increased by the same factor.

Perfect elastic collisions: For value-pairs on the stability boundaries the amount of energy generated is exactly zero. Thus, these valuepairs can be used for realizing perfect elastic collisions. Yet, as the virtual damping force is susceptible to measurement or discretization errors (the velocity is the derivation of the position) especially the lower half of the stability boundaries is suited for realizing elastic collisions. For small values equation (7) and (8) can be used for this task.

Perfect plastic collisions: With plastic collisions the velocity must vanish without changing its sign. These prerequisites can be guaranteed inside region $A$.

This paper addresses the control theory, but the human perception of virtual collision is not considered yet. To achieve a realistic impression of haptic simulations, this issue may not be underestimated and must be examined in the future. Another open issue is the exact effect of a mechanical damping and a larger time delay on the stability boundary.

\section{REFERENCES}

Basdogan, C. and M. A. Srinivasan (2001). Haptic rendering in virtual environments. In: Virtual Environments HandBook (K. Stanney, Ed.). Lawrence Erlbaum Associates, Inc.. pp. 117-134.

Colgate, J.E. and J.M. Brown (1994). Factors affecting the z-width of a haptic display. In: Proc. of the IEEE Int. Conf. on Robotics and Automation. pp. 3205-3210.

Franklin, G.F., J.D. Powell and M.L. Workman (1998). Digital Control of Dynamic Systems. third ed.. Addison-Wesley. Menlo Park.

Gil, J.J., A. Avello, Á. Rubio and J. Flórez (2004). Stability analysis of a 1 dof haptic interface using the routh-hurwitz criterion. IEEE Transactions on Control Systems Technology pp. 583-588.

Massie, T.H. and J.K. Salisbury (1994). The phantom haptic interface: A device for probing virtual objects. In: Proc. of the ASME Winter Annual Meeting, Symposium on Haptic Interfaces for Virtual Environment and Teleoperator Systems. Chicago. pp. 295-302.

R. J. Adams and B. Hannaford (1999). Stable haptic interaction with virtual environments. IEEE Trans. on Robotics and Automation 15(3), 465-474.

Salcudean, S.E. and T.D. Vlaar (1997). On the emulation of stiff walls and static friction with a magnetically levitated input/output device. ASME Journal of Dynamic Systems, Measurement and Control. 